\title{
AN ALGEBRAIC SOLUTION OF THE EINSTEIN EQUATIONS*
}

BY

\author{
EDWARD KASNER
}

The Einstein field equations of gravitation in their cosmological form (for the case where matter is not present) may be written

$$
R_{i k}-\lambda g_{i k}=0
$$

or, what is equivalent,

$$
R_{i k}-\frac{1}{4} g_{i k} R=0
$$

We wish to present here a new particular solution which is algebraic and extremely simple both in analytic and geometric form, namely

$$
d s^{2}=x_{1}^{-2}\left(d x_{1}^{2}+d x_{2}^{2}\right)+x_{8}^{-2}\left(d x_{8}^{2}+d x_{4}^{2}\right) .
$$

It is in fact the simplest solution beyond the hypersphere (De Sitter's solution)

$$
d s^{2}=x_{1}^{-2}\left(d x_{1}^{2}+d x_{2}^{2}+d x_{3}^{2}+d x_{4}^{2}\right) .
$$

Our starting point is to assume the quaternary form

$$
y_{i k} d x_{i} d x_{k}
$$

to be the sum of two binary forms, one in the variables $x_{1}, x_{2}$, the other in the variables $x_{3}, x_{4}$.

We have then

(5) $d s^{2}=E d x_{1}^{2}+2 F d x_{1} d x_{2}+G d x_{2}^{2}+E^{\prime} d x_{3}^{2}+2 F^{\prime} d x_{3} d x_{4}+G^{\prime} d x_{4}^{2}$

where $E, F, G$ involve only $x_{1}, x_{2}$ and $E^{\prime}, F^{\prime}, G^{\prime}$ involve only $x_{3}, x_{4}$. By a transformation of variables we may, without loss of generality, assume

* Presented to the Society, October 29, 1921, and to the National Academy of Sciences, April, 1921. 
so that

$$
\begin{array}{ll}
F=0, & E=G=\mu\left(x_{1}, x_{2}\right), \\
F^{\prime}=0, & E^{\prime}=G^{\prime}=v\left(x_{3}, x_{4}\right)
\end{array}
$$

$$
d s^{2}=\mu\left(x_{1}, x_{2}\right)\left(d x_{1}^{2}+d x_{2}^{2}\right)+\nu\left(x_{8}, x_{4}\right)\left(d x_{8}^{2}+d x_{4}^{2}\right) .
$$

The problem is to find the two functions $\mu$ and $\nu$ so that equations (2) are satisfied. It is convenient to introduce

$$
\alpha=\frac{1}{2} \log \mu, \quad \beta=\frac{1}{2} \log \nu
$$

The components of the contracted curvature tensor are easily found to be*

$$
\begin{aligned}
& R_{11}=R_{22}=\alpha_{11}+\alpha_{28}, \\
& R_{33}=R_{44}=\beta_{33}+\beta_{44},
\end{aligned}
$$

where subscripts applied to $\alpha$ and $\beta$ denote partial derivatives $\alpha_{11}=\partial^{2} \alpha / \partial x_{1}^{2}$, etc. The other components $R_{12}$, etc., vanish identically.

The scalar curvature is

$$
R=2 \frac{\alpha_{11}+\alpha_{22}}{\mu}+2 \frac{\beta_{38}+\beta_{44}}{\nu} .
$$

Substituting in (2) we find merely one condition, which may be written

$$
\frac{\alpha_{11}+\alpha_{22}}{\mu}=\frac{\beta_{33}+\beta_{44}}{\nu}
$$

We observe that the left member is a function of $x_{1}, x_{2}$ and the right member is a function of $x_{3}, x_{4}$, therefore both members are equal to a constant, that is, using $\left(6^{\prime}\right)$,

$$
e^{-2 \alpha}\left(\alpha_{11}+\alpha_{28}\right)=c, \quad e^{-2 \beta}\left(\beta_{33}+\beta_{44}\right)=c .
$$

This means that the curvature of each of the two binary forms (surfaces) is constant. It follows that each may be assumed as the first fundamental quadratic form of a sphere (radius $=c^{-1 / 2}$ ). It is, therefore, unnecessary

* We may conveniently apply the formulas given on p. 229 of the author's papes The solar gravitational field completely determined by its light rays, Mathematische Annalen, vol. 85 (1922), pp. 227-236. 
actually to write out the general solutions of the above partial differential equations. It is sufficient to assume the particular solution

$$
2 \alpha=\log \frac{1}{c x_{1}^{2}}, \quad 2 \beta=\log \frac{1}{c x_{8}^{2}} .
$$

Our quaternary form (6) is thus

$$
d s^{2}=\frac{d x_{1}^{2}+d x_{2}^{2}}{c x_{1}^{2}}+\frac{d x_{8}^{2}+d x_{4}^{2}}{c x_{8}^{2}} .
$$

If the constant $c$ is zero we may take $\alpha=0, \beta=0$, that is, $\mu=1$, $\nu=1$, so that

$$
d s^{2}=d x_{1}^{2}+d x_{2}^{2}+d x_{3}^{2}+d x_{4}^{2},
$$

which is euclidean 4-space. Excluding this trivial case every solution of our problem is equivalent to (3) under a homothetic transformation.

If the sum of two independent binary forms is to satisfy (2) then the forms represent equal spheres and the result is reducible to (3).

Using another familiar element of a sphere we may write the form (which is equivalent to (7) with $c=4 a$ )

$$
d s^{2}=\frac{d x_{1}^{2}+d x_{2}^{2}}{\left[1+a\left(x_{1}^{2}+x_{2}^{2}\right)\right]^{2}}+\frac{d x_{8}^{2}+d x_{4}^{2}}{\left[1+a\left(x_{8}^{2}+x_{4}^{2}\right)\right]^{2}} .
$$

Similarly the four-dimensional hypersphere (4) may also be written in the form

$$
d s^{2}=\frac{d x_{1}^{2}+d x_{2}^{2}+d x_{8}^{2}+d x_{4}^{2}}{\left[1+a\left(x_{1}^{2}+x_{2}^{2}+x_{8}^{2}+x_{4}^{2}\right)\right]^{2}} .
$$

Thus (8) is an exact solution of the field equations (2) as well as (8').

We may interpret our result geometrically in space of six dimensions as follows.

Take a flat space with six cartesian coordinates $X_{1}, X_{2}, X_{3}, X_{4}, X_{5}, X_{6}$. In the 3-flat $X_{1}, X_{2}, X_{3}$, take a unit sphere

$$
X_{1}^{2}+X_{2}^{2}+X_{3}^{2}=1,
$$

and in the 3-flat $X_{4}, X_{5}, X_{6}$, another unit sphere

$$
X_{4}^{2}+X_{6}^{2}+X_{6}^{2}=1 \text {. }
$$


On these spheres as bases construct hypercylinders of five dimensions. The equations of these cylinders are $\left(9^{\prime}\right)$ and $\left(9^{\prime \prime}\right)$. The intersection of these cylinders is the four-dimensional manifold defined by the simultaneous equations

$$
X_{1}^{2}+X_{2}^{2}+X_{8}^{2}=1, \quad X_{4}^{2}+X_{5}^{2}+X_{6}^{2}=1 .
$$

This is an algebraic four-dimensional manifold of fourth degree which obeys the field equations (2).

Any four-dimensional manifold in a 6 -flat which is the intersection of two cylindrical 5-spreads,

$$
F\left(X_{1}, X_{2}, X_{3}\right)=0, \quad G\left(X_{4}, X_{5}, X_{6}\right)=0,
$$

will have for its $d s^{2}$ a quaternary form which can be written as the sum of two independent binary forms. Thus we have proved

The only manifolds of type (11) in flat space of 6 dimensions which obey the field equations are reducible to the quartic variety (10).

\section{SEPARABLE FORMS}

A quadratic differential form in $n$ variables may be called separable if it can be reduced by any transformation of the variables to the sum of two forms one involving $h$ variables and the other involving $k$ variables, where $h+k=n$.

This we shall call separable of type $(h, k)$. The various types for a given $n$ are not necessarily mutually exclusive. There are possibilities of certain special forms belonging to more than one type. We may of course also have separable forms of type $\left(h_{1}, h_{2}, \cdots, h_{r}\right)$, where $h_{1}+h_{2}+\cdots+h_{r}=n$. If the form is euclidean it is of type $(1,1, \ldots, 1)$, and vice versa this type is obviously euclidean. This is the extreme case of separability, the $n$-ary form being then transformable into the sum of $n$ independent unary forms.

If $n=4$, the possible types are

(a) $(2,2)$,

(b) $(3,1)$,

(c) $(2,1,1)$,

(d) $(1,1,1,1)$.

When can an Einstein manifold be of one of these types? For $(a)$ the result has been given above. For $(b)$ no solutions exist; the proof is easy but is here omitted.

For (c) no solutions exist except of course those which are of the trivial euclidean type $(d)$. Hence we have the theorem 
If an Einstein manifold is to be separable it must be either euclidean or equivalent to (3), that is the quartic manifold (10).

The separability so far defined has been complete. There is another more general theory of incomplete or partial separability. Thus a form in four variables $x_{1}, x_{2}, x_{3}, x_{4}$ may in certain cases, even though not separable in the first sense, be reducible for example to the sum of three binary forms

$$
d s^{2}=Q^{\prime}+Q^{\prime \prime}+Q^{\prime \prime \prime}
$$

where $Q^{\prime}$ involves $x_{1}, x_{2}, Q^{\prime \prime}$ involves $x_{1}, x_{3}$, and $Q^{\prime \prime \prime}$ involves $x_{1}, x_{4}$. This refers of course to the coefficients as well as the differentials, that is

$$
Q^{\prime}=g_{11} d x_{1}^{2}+2 g_{12} d x_{1} d x_{2}+g_{22} d x_{2}^{2},
$$

where the $g$ 's are functions of only $x_{1}, x_{2}$. Einstein solutions of type (12) actually exist. In particular I have found all solutions of the form*

$$
d s^{2}=\alpha\left(x_{1}\right) d x_{1}^{2}+\beta\left(x_{1}\right) d x_{2}^{2}+\gamma\left(x_{1}\right) d x_{8}^{2}+\delta\left(x_{1}\right) d x_{4}^{2} .
$$

These are included in the type (12). They may be immersed in a flat space of seven dimensions and defined in finite form by means of three surfaces of rotation having a common axis.

* See Science, vol. 54 (1921), p. 304, and American Journal of Mathematics, vol. 43 (1921), p. 220; also a forthcoming paper in these Transactions.

Columbia University,

NEW YoRK, N. Y. 